\title{
PET Studies of the Effects of ECT on Cerebral Physiology
}

\author{
M. Nobler, S. Yu, B. Mensh, S. Lisanby, L. Alkalay, R. Van Heertum, E. Heyer, \\ H. Sackeim
}

Columbia University, New York, USA

\section{Introduction}

ECT is a remarkably effective treatment for major depressive disorder [MDD]. Neuroimaging studies of rCBF and rCMR are helpful in addressing mechanisms of therapeutic effects. We have reported that reductions in $\mathrm{rCBF}$ in prefrontal regions are associated with positive response to ECT[1], and that a course of bilateral ECT is associated with marked reduction in prefrontal rCMR[2]. We now report on a new study of patients receiving ECT for MDD. This study provides the first comparison of the relative acute effects of seizures on both $\mathrm{rCBF}$ and $\mathrm{rCMR}$.

\section{Methods}

Fourteen inpatients met research diagnostic criteria for MDD. Each patient underwent positron emission tomography [PET]: (1) PreECT [following medication washout]: $1 \mathrm{rCBF}\left[{ }^{15} \mathrm{O}-\mathrm{H}_{2} \mathrm{O}\right]$ and $1 \mathrm{rCMR}\left[{ }^{18} \mathrm{~F}\right.$-deoxyglucose; FDG] scan; (2) during ECT \#3: rCBF scan just prior to ECT, rCBF scans at 20 and $40 \mathrm{~min}$ in the postictal state, rCMR scan at 50 min postictal; (3) 2-7 days post ECT course [medication free]: $1 \mathrm{rCBF}$ and $1 \mathrm{rCMR}$ scan. Prior to radioisotope injection $\left[40 \mathrm{mCi}{ }^{15}\right.$ or $5 \mathrm{mCi}$ FDG], a catheter was placed in the left radial artery for arterial sampling for full quantification. Scans were quantified using the autoradiographic method[3]. Scans were acquired with a Siemens ECAT HR+ scanner [63 slices, 4.2-5.4 mm FWHM] in $2 \mathrm{D}$ mode for $\mathrm{rCBF}$ and $3 \mathrm{D}$ mode for $\mathrm{rCMR}$. Images were reconstructed using attenuation correction measured by a 15 -min transmission scan.

We conducted two separate analyses: (1) After application of an anisotropic-diffusion filter, T1-weighted MRIs were segmented with a thresholding procedure and grossly parcellated into the following broad areas: CSF, cortical gray and white matter, subcortical gray matter, midbrain, pons, and cerebellum. The PET scans were registered to the MRIs. rCBF values for each of the 6 regions [excluding CSF] were determined using partial volume correction. (2) We also conducted a preliminary Statistical Parametric Mapping[4] [SPM-99] analysis of the changes in rCBF that occurred between the preECT baseline and the first ${ }^{15} \mathrm{O}$ measurement in the postictal state, using a corrected cluster size probability of $\mathrm{P}<0.001$ [filter $=12$ ]. We conducted the same analysis on the FDG data collected at baseline and 50 min into the postictal state, using a cluster size probability of only $\mathrm{P}<0.01$ [filter $=12$ ].

\section{Results}

Across a subgroup of the patients, ECT led to marked postictal reductions in fully quantified global cortical $\mathrm{rCBF}[\mathrm{P}<0.001]$, with little impact in subcortical regions. 
The cortical effects were most pronounced 20-40 min post ECT, but persisted during the week post ECT. No consistent changes were seen within the broad categories of subcortical gray, midbrain, pons, cerebellum or white matter at either time point.

SPM data on 12 patients are available and revealed marked $\mathrm{rCBF}$ reductions in at the prefrontal pole and dorsolateral PFC, medial PFC, and parietal cortex [P's $<.0001]$. Clusters of significant $\mathrm{rCBF}$ reductions are represented in Figure 1. We conducted the same analysis on the FDG data collected at baseline and $50 \mathrm{~min}$ into the postictal state. This is represented in Figure 2. The pattern of significant rCMR reductions mirrored the $\mathrm{rCBF}$ changes, but the magnitude of rCMR reductions was much less marked [as indicated by the cluster size probability of only $\mathrm{P}<.01]$. All increases in rCBF and rCMR occurred in posterior, occipital areas, suggesting a change in the anterior/posterior gradient and the increases reflecting the artifact that SPM does not account for absolute changes. Highly similar effects were observed when the resting rCBF scan conducted just prior to the treatment was compared to the first postictal scan, indicating that the postictal state was associated with marked topographic change. The divergence between $\mathrm{rCBF}$ and $\mathrm{rCMR}$ data indicates that ECT may result in uncoupling between cerebral perfusion and metabolic rate in the postictal state, with greater reductions in blood flow than metabolism.
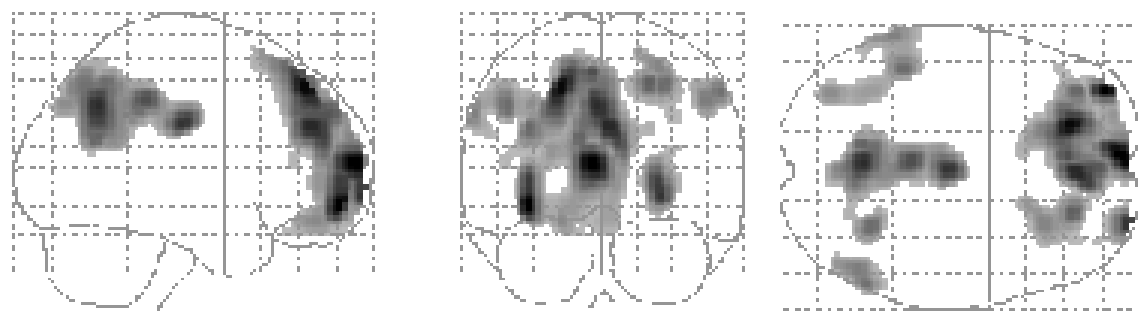

Figure 1. SPM Results for Acute Effects on rCBF Relative to preECT Baseline
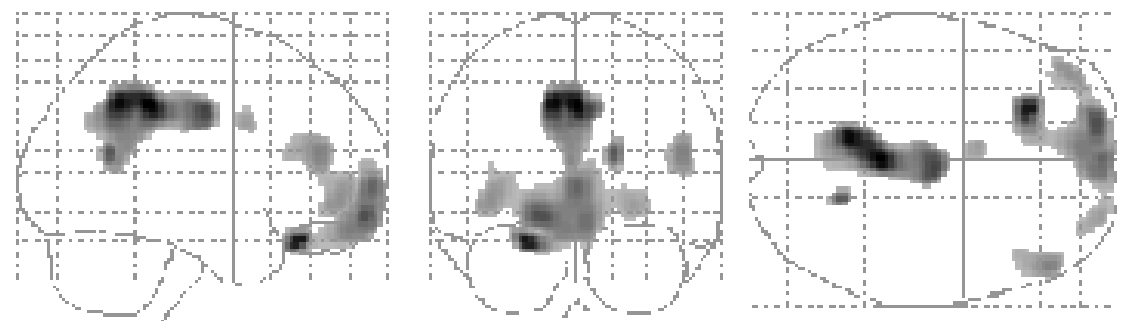

Figure 2. SPM Results for Acute Effects on rCMR Relative to preECT Baseline

\section{Conclusions}

The data thus far suggest that the $\mathrm{rCBF}$ and $\mathrm{rCMR}$ reductions in the immediate postictal state and following the ECT course are most marked in the neocortex, with inconsistent change when subcortical regions are treated as a single ROI. However, verification of these initial findings for views regarding the centrality of subcortical [eg, diencpehalic] structures for the efficacy of ECT, as well as the possibility of 
uncoupling of $\mathrm{rCBF}$ and $\mathrm{rCMR}$ in the immediate postictal period, must await fine grained ROI and voxel-based analyses, in particular implementing methods for full brain parcellation [ROI determination] and subsequent partial volume correction.

\section{References}

1. Nobler, M. S., Sackeim, H. A., Prohovnik, I., Moeller, J. R., Mukherjee, S., Schnur, D. B., Prudic, J., and Devanand, D. P.: Regional cerebral blood flow in mood disorders, III. Treatment and clinical response. Arch Gen Psychiatry 51 (1994) 884-97

2. Nobler, M. S., Oquendo, M. A., Kegeles, L. S., Malone, K. M., Campbell, C., Sackeim, H. A., and Mann, J. J.: Decreased regional brain metabolism following electroconvulsive therapy. Am J Psychiatry 158 (2001) 305-308

3. Raichle, M. E., Martin, W. R., Herscovitch, P., Mintun, M. A., and Markham, J.: Brain blood flow measured with intravenous H2(15)O. II. Implementation and validation. J Nucl Med 24 (1983) 790-798.

4. Friston, K. J., Holmes, A. P., Worsley, K. J., Poline, J. B., Frith, C. D., and Frackowiak, R. S. J.: Statistical parametric maps in functional imaging: A general approach. Hum Brain Mapping 2 (1995) 189-210 